\title{
Experimental set-up and the associated model for squeal analysis
}

\author{
Martin Duboc ${ }^{1,2,3}$, Vincent Magnier ${ }^{1,2,3, *}$, Jean-François Brunel ${ }^{2,3}$, and Philippe Dufrénoy ${ }^{1,2}$ \\ ${ }^{1}$ Université Lille Nord de France, 59000 Lille, France \\ ${ }^{2}$ Lille-LaMcube, 59655 Villeneuve d'Ascq, France \\ ${ }^{3}$ CNRS, FRE 2016, 59655 Villeneuve d'Ascq, France
}

Received: 21 May 2019 / Accepted: 6 November 2019

\begin{abstract}
Brake squeal is commonly defined at frequency upper than $1000 \mathrm{~Hz}$ and occurs if the system has a very high amplitude mechanical vibration with sound pressure level above $120 \mathrm{~dB}$. Many studies are devoted to this problem and many of recent ones show that contact conditions and friction material behavior have a major influence on squeal occurrence. To investigate this aspect, an experimental set-up has been developed in this study. It is based on a simplified system in order to focus on the influence of the material in the one hand and surface conditions in the other hand. In this paper, the design of the pin-on-disc is described and an analytical model is also presented in order to understand the dynamic behavior of the system. Macroscopic aspects are investigated by varying the pin geometry. The results show clearly the influence of the variation of the contact length size on squeal occurrence. Comparison with the model shows good agreement and exhibit the necessity of considering an improved model of the friction material behavior. This study also gives information on the comprehension of squeal mechanisms.
\end{abstract}

Keywords: Brake squeal / friction material/ experimental / numerical comparison

\section{Introduction}

It is commonly accepted by the scientific community that self-excited vibrations initiated by frictional forces can lead to noise in brake systems. This is particularly true for the squeal, which is characterized by high frequencies $(1-10 \mathrm{kHz})$ and high sound pressure levels of up to $120 \mathrm{~dB}$ [1]. Even though the reliability of the system itself is unaffected, the squeal phenomenon is annoying to users and remains one of the major challenges faced by the industry of transportation. Although there are many available models for predicting the dynamic behavior of brake systems, the conditions for the occurrence of such self-excited vibrations are still not fully understood. On a global point of view, it is now assumed that squeal is linked to a mode lock-in corresponding to self-excited vibrations when system frequencies merge [2-4]. More precisely, the natural modes of the system vary with the contact loading and coalesce with contact condition, thereby leading to unstable modes. Typical squeal frequency spectra show one or more fundamentals closely associated with disc natural frequencies and their harmonics showing that pad constraining effects are not excessive enough to alter the mode shapes. During braking, self-excited vibrations may be triggered by favorable pressure, sliding velocity, temperature and humidity conditions. Despite this understanding,

\footnotetext{
*e-mail: vincent.magnier@polytech-lille.fr
}

brake squeal still appears as a hazardous phenomenon because it is affected by many factors. Even if the mode lock-in appears as necessary conditions, influencing parameters need to be considered. It is important to bear in mind that the contact at the friction interface is not perfect and is a very complex tribological situation with non smooth surfaces, material transformation beneath the contact, third body layer of wear particles accumulation, etc. [5-7]. Recent works have shown that the evolution of the friction material, surfaces and tribolayer, with a braking sequence, could influence the activation of squeal occurrence [8]. In the experiments of [9] it is, for example, shown that surface topology evolution and material transformation beneath the surface lead to the emergence of squeal of an automotive disc brake system. However, the system is very complex and it is difficult to identify which parameter is relevant. In other works, focused on friction material evolution and tribology, it is shown that the situation is complex as the friction material is modified by the thermal, mechanical and tribological loading as far as the tribolayer [10-12]. In order to highlight the role of these parameters, one way is to investigate on a reduced set-up with a simplified and well identified dynamic behavior, with squeal occurrence which could be influenced by an evolution of material and tribolayer.

In terms of modeling, many works have been done, with complex or reduced models. The last ones are generally developed to explain the system dynamic phenomena as 


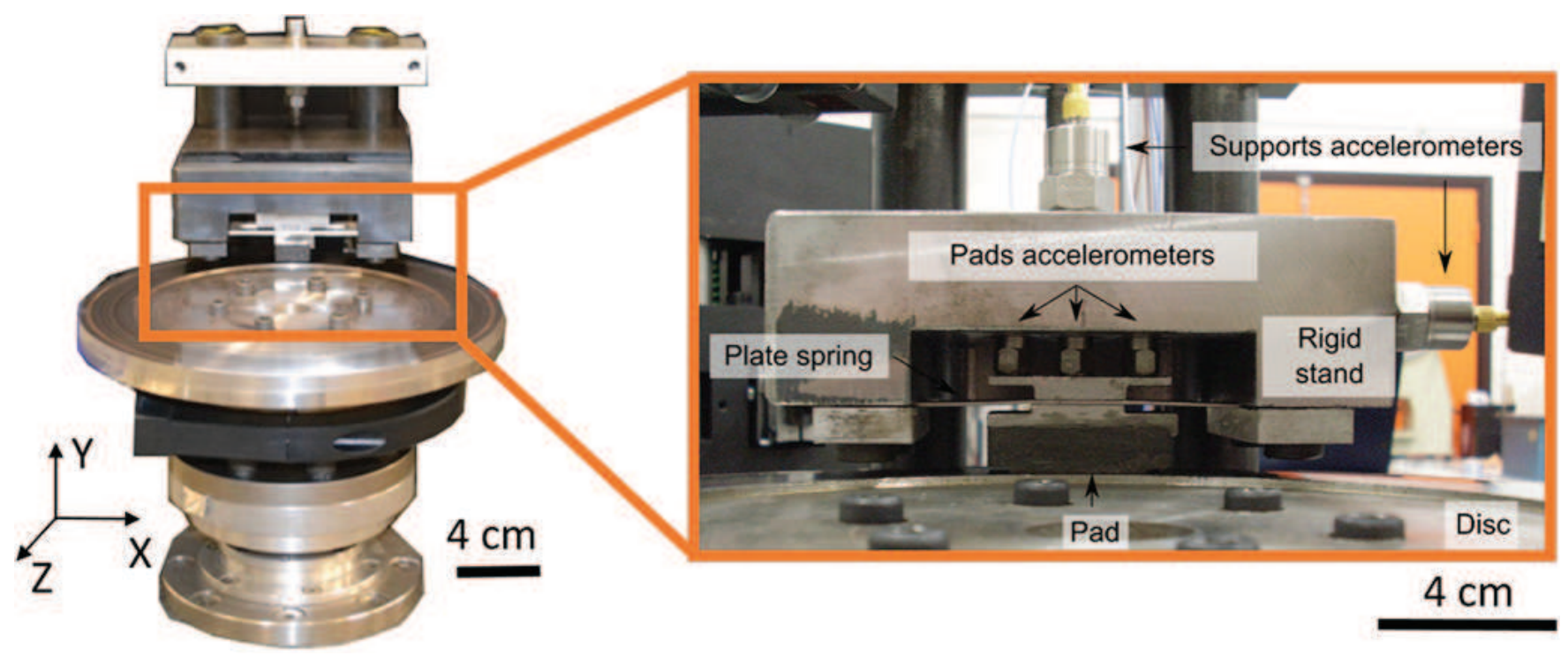

Fig. 1. Experimental set-up.

mode-coupling by Hoffmann and Gaul with a two degrees of freedom model [6]. Ouyang et al. [13] investigated the effects of stiffness and damping variation by means of a complex modal analysis applied to a simplified model.

More complex models, introducing the description of components and connections, boundary and loading conditions help to identify more quantitatively each system parameter on instabilities. Triches et al. [14] used a finite element model to study the effects of varying the friction coefficient, temperature, braking pressure, wear and material properties. For example, they demonstrated that an increase in temperature modifies the contact stiffness and potentially reduces the propensity for brake squeal. Dai et al. [15] carried out a numerical investigation of the effect of adding chamfers to pad linings and found that chamfering lowers the propensity for instability. More recent works introduce enriched material and surface parameters and their evolution with loading, showing as in experiments, that they could influence the instability [16]. Reference [17] propose a numerical strategy which allows to integrate the roughness at the interface into a complete brake with a squeal study. They show that the roughness is important to consider. At this scale with an experimental point of view, [18] show that the roughness in the contact could be a factor for reducing or suppressing squeal. From a point of view more macroscopic always in modeling, [19] aims to take different pads position in the process of the prediction of squeal noise and to investigate the sensitivity of the squeal propensity to these various contact interfaces.

The present work is in the way of investigating the effect of friction material evolution and tribolayer on squeal occurrence. To investigate this aspect, it is proposed to develop a dedicated experimental set-up. It is based on a simplified system in order to focus on the influence of the material pair and surface conditions. The design of the set-up is described and an analytical model is also presented in order to understand the dynamic behavior of the system. Macroscopic aspects are investigated by varying the pin geometry and loading conditions (pressure and sliding velocities). With the dedicated instrumentation and the numerical model, the results of squeal occurrence and frequencies can be understood, as far as some parameters which are important to be considered.

\section{Squeal experimental set-up}

\subsection{Presentation of the pin-on-disc}

The idea was to design a system reaching squeal frequency included in a range from 2 to $12 \mathrm{kHz}$ in agreement with frequency level observed in brakes, but with a controlled dynamic behavior. Several simplified experimental set-ups have been proposed in the bibliography. Massi et al. [20] developed an experimental set-up to investigate the mode lock-in phenomenon $[6,15]$. Oura et al. designed a bench to investigate the influence of the contact stiffness on the brake squeal occurrence [7]. Figure 1 presents the simplified pin-on-disc architecture designed in this paper for the experimental set-up. It is in the spirit of the system developed by Oura et al. The bench is made of a disc and a pin fixed on a flexible plate clamped in a rigid stand.

The disc is made of C45 steel and its boundary conditions are inner fixed and outer free. The outer diameter is $215 \mathrm{~mm}$ and the thickness of the friction track is $15 \mathrm{~mm}$. The dimensions have been chosen to obtain eigenfrequencies in the range of several $\mathrm{kHz}$ as for a braking system. The pad, made of friction material, is fixed on the center of a thin plate acting as a spring, and the extremities of this plate are fixed on a rigid stand. The thickness of the lame is $2 \mathrm{~mm}$. The pad dimensions are $40 \mathrm{~mm}$ length (sliding direction), name as "pad length" in the following, $20 \mathrm{~mm}$ width and $10 \mathrm{~mm}$ thickness. The translation displacement of the rigid stand is controlled to generate the thrust load. The pad is located at $95 \mathrm{~mm}$ along the 


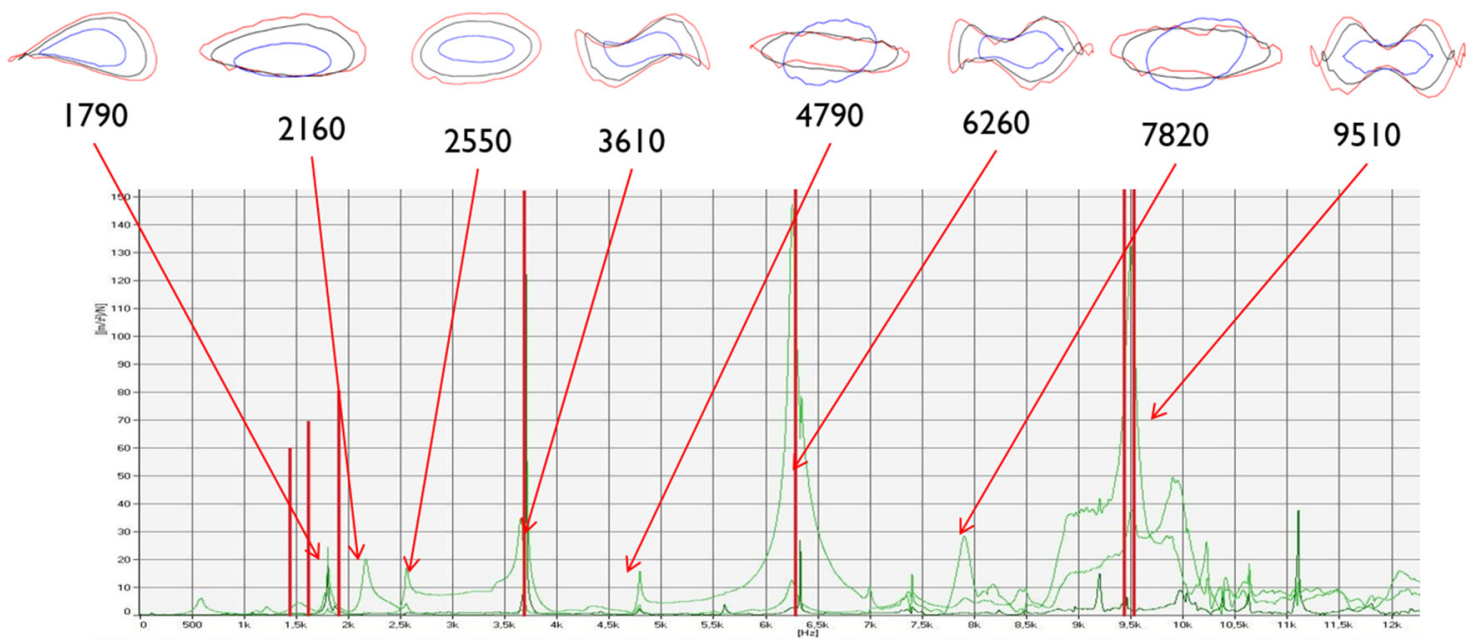

Fig. 2. FRF of the set-up obtained from impact hammer tests (disc without contact with the pin and fixed on the tribometer.

radius from the rotational center of the disc. For the tests, the instrumentation is equipped of a microphone located near the disc surface $(100 \mathrm{~mm})$ measuring the generated squeal. Accelerometers are located on the pin housing at the trailing edge, on the middle and at the leading edge. A torque-meter is inserted between the disc and the rotor. The thin plate is instrumented with two half-bridge gauges, one at the leading edge side of the pad and one at the trailing edge. These gauges record the deformation of the static bending deformation of the plate which has been calibrated to determine the corresponding normal force during the test. Two accelerometers have been added on the rigid stand to control the low level of vibrations on this component.

\subsection{Determination of set-up eigenfrequencies}

First, the experimental frequencies of the disc and the pad-caliper assembly have been determined without discpad contact using impact hammer and acceleration measurements. On the disc, the mesh allowing the positioning of the triaxial accelerometer is composed of 3 circles and 48 radius for a total of 144 points. Figure 2 shows the frequency response function FRF with the usual notation $(n, m)$ of the deformed shape $\mathrm{n}$ is the number of nodal diameter and $\mathrm{m}$ is the number of nodal circle. The identified disc frequencies refer to modes with nodal diameters due to the experimental configuration (out-ofplane impact and out-of-plane acceleration measurements on $\mathrm{x}$ points on the circumference and $\mathrm{y}$ points along the radius). For the pin, the accelerometers have been used in order to identify the tilting mode by phase measurements between the two accelerometers located along the pin in the friction direction, at the leading and the trailing edges. Table 1 shows the two first eigenfrequencies of the pin-plate assembly without contact with the disc.

Figure 3 shows the eigenfrequencies of the system with contact between pin and disc and obtained by the same impact hammer procedure as done for the disc. The frequencies are close to the disc natural ones as the pad constraining effects are not excessive enough to alter the
Table 1. Eigenfrequencies of the pin-plate assembly obtained from impact hammer tests without contact with the disc.

\begin{tabular}{lll}
\hline Pad mode & 1st bending \\
\hline Frequency $(\mathrm{Hz})$ & $637 \mathrm{~Hz}$ & 2nd bending \\
\hline
\end{tabular}

mode shapes. Accelerometers on the pin housing exhibit the second bending shape of the pin-plate assembly which is associated with the lowest frequency at $1750 \mathrm{~Hz}$ considering a reduced contact length of $10 \mathrm{~mm}$ (this parameter is studied later in Sect. 2.3). This is due to the combination of the deformed shapes of the disc and pad with contact. In other words at low frequency, the disc exhibits 2 waves with relatively large amplitude so that the pin-plate assembly is preferably deformed as its second bending shape to suit the disc deformed shape. The pin deformed shape is harder to detect at a higher frequency as the amplitudes are lower. It could be expected that the first bending pin-lame assembly mode shape could be obtained at a higher frequency for higher wave number and lower amplitude of the disc deformation.

\subsection{Test protocol and geometry of the pin}

Before the test the surface of the pin is polished. The system is initially loaded before the disc is rotated. First, a $0.25 \mathrm{~mm}$ displacement of the rigid stand is applied, corresponding to a load of $500 \mathrm{~N}$ and an average pressure of $0.625 \mathrm{MPa}$ for a contact area of $40 \times 20 \mathrm{~mm}^{2}$. Before each test, the contact surface between the disc and the pin is measured to control the contact uniformity along the pin length.

A constant velocity of $20 \mathrm{rpm}$ is secondly applied, corresponding to a linear velocity of $0.1 \mathrm{~m} / \mathrm{s}$ on the mean radius. The test is made of several tests of $50 \mathrm{~min}$. 


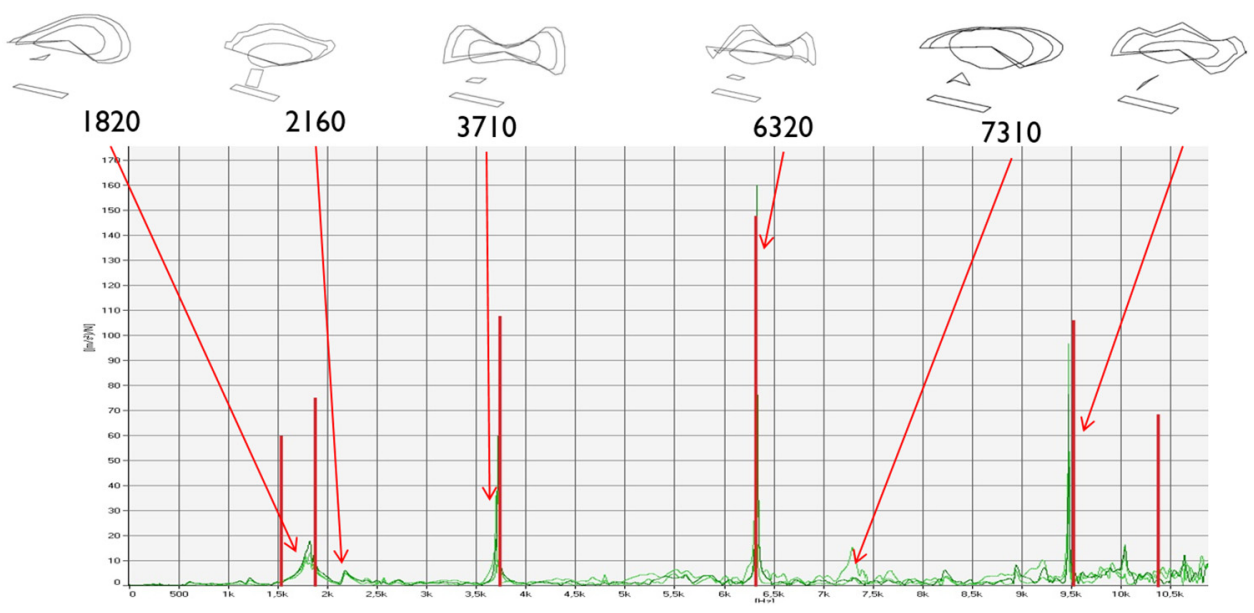

Fig. 3. FRF and mode shape of the experimental set-up with contact between the disc and the pin.

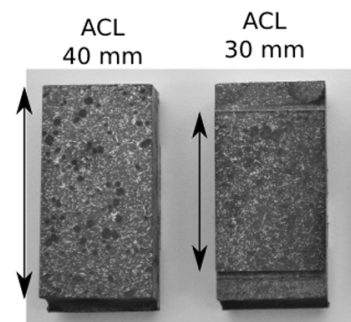

$\mathrm{ACL}$ $20 \mathrm{~mm} \quad 10 \mathrm{~mm}$

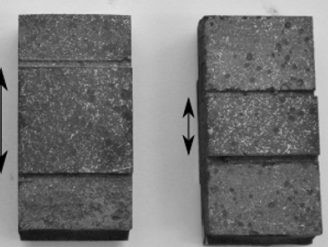

(a)

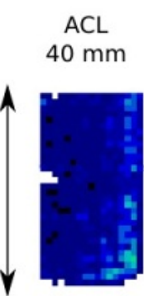

$\mathrm{ACL}$

$30 \mathrm{~mm}$

$\mathrm{ACL}$

$20 \mathrm{~mm}$

$\mathrm{ACL}$
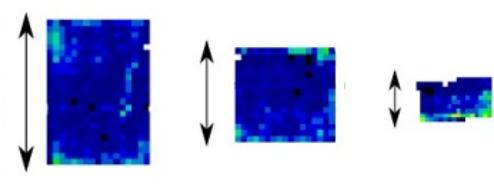

(b)
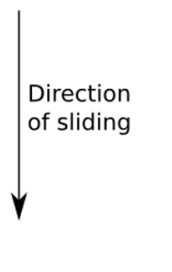

Direction of sliding

Fig. 4. (a) Friction pin for the different ACL. (b) Cartography for each ACL for a normal force of $400 \mathrm{~N}$.

The tests have been done for different pin lengths of 40, 30, 20 and $10 \mathrm{~mm}$, named ACL for Apparent Contact Length. The pin width is always $20 \mathrm{~mm}$. In order to not modify the global dynamic behavior of the pin, the mass and dimensions of the pin are modified as low as possible for each pad dimension, by machining only a height of $1 \mathrm{~mm}$ to obtain the reduced ACL (Fig. 4a). For this study, a reduced formulation friction pad is developed. Here, friction pad has only six components (Resin, Baryte, Twaron fibers, Steel fibers, Mineral fibers, Graphite). This material is developed in collaboration with the company FLERTEX. Figure 4b shows the static contact pressure distribution measured with pressure film sensor before the test. Black areas correspond to a zero pressure and bright ones to maximum intensity. Uniformity of the contact is difficult to obtain but the test is started as soon as the contact is obtained on the full area.

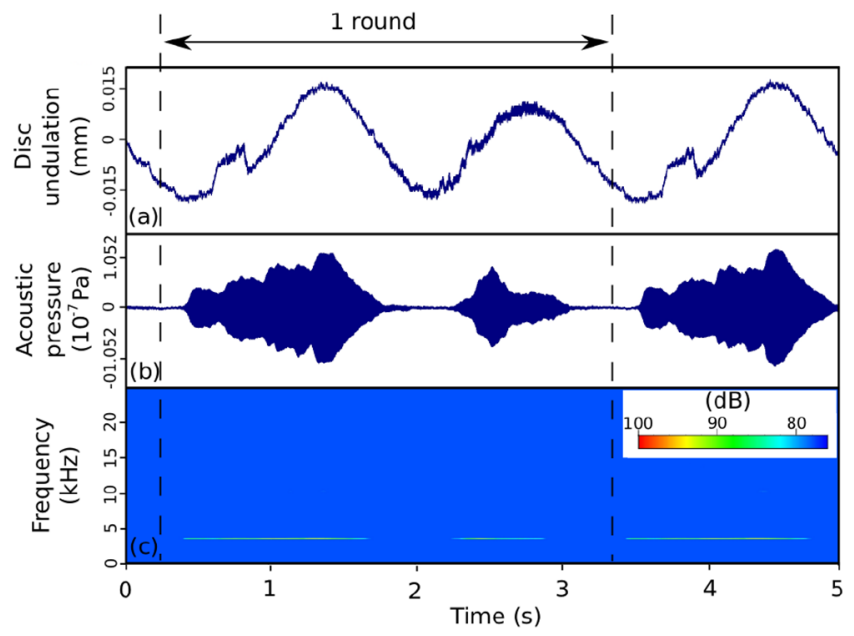

Fig. 5. Results from test with the pin of ACL $20 \mathrm{~mm}$ : (a) raw signal of disc waviness, (b) raw signal of acoustic pressure, (c) time-frequency diagram of the signal of acoustic pressure.

\subsection{Experimental results}

Figure 5 a shows the raw signal of the disc waviness during the last test of $5 \mathrm{~s}$ for the pin of $20 \mathrm{~mm}$ length. It can be noticed that the waviness is about $30 \mu \mathrm{m}$. Figure $5 \mathrm{~b}$ presents a raw acoustic pressure signal measured by the microphone exhibiting that the noise occurrence correlates with a disc elevation. The squeal frequency is around $3400 \mathrm{yHz}$ with a sound pressure level around $100 \mathrm{~dB}$.

Acoustic emissions are obtained for all configurations with sound pressure level reaching $100 \mathrm{~dB}$. Figure 6 summarizes the results for the four configurations and the long duration test. Each point corresponds to an occurrence of noise higher than $100 \mathrm{~dB}$, the track being made every 5 minutes. In this figure, friction coefficient and squeal frequencies can be seen. The main difference between all the configurations is the frequencies involved during the squeal period. For each configuration, a variation of friction coefficient is obtained with values comprise between 0.2 and 0.4 . The frequencies strongly depend on 
Table 2. Squeal frequencies for all ACL configurations.

\begin{tabular}{lllll}
\hline ACL $(\mathrm{mm})$ & $40 \mathrm{~mm}$ & $30 \mathrm{~mm}$ & $20 \mathrm{~mm}$ & $10 \mathrm{~mm}$ \\
\hline Squeal frequencies $(\mathrm{Hz})$ & $\mathbf{7 8 0 0}$ & $2600 / \mathbf{6 0 0 0}$ & $\mathbf{3 4 0 0} / 6800$ & $\mathbf{1 6 0 0}$ \\
\hline
\end{tabular}

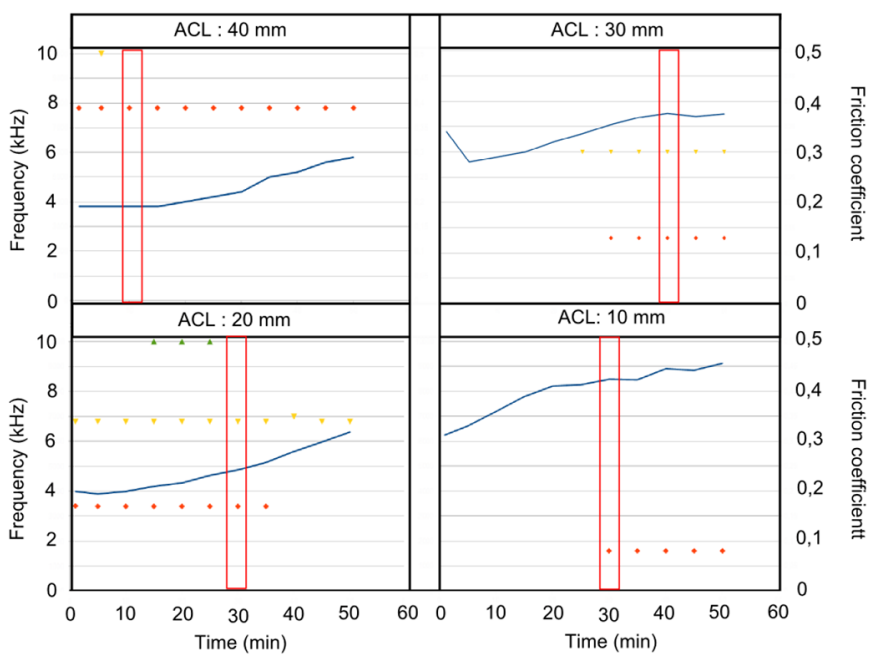

Fig. 6. Experimental results for all ACL configurations (a) $40 \mathrm{~mm} \mathrm{(b)} 30 \mathrm{~mm} \mathrm{(c)} 20 \mathrm{~mm}$ (d) $10 \mathrm{~mm}$, points: squeal frequencies; blue curve: friction coefficient.

the contact length. Table 2 shows the associated squeal frequencies with each ACL. Note that squeal frequencies are closed to the ones obtained without contact, allowing identifying the disc mode shape involved. A squeal frequency of $2600 \mathrm{yHz}$ is obtained for $\mathrm{ACL}=30 \mathrm{~mm}$ but it has been identified as a machine spindle eigenfrequencie by separated investigations on the spindle [21]. For $\mathrm{ACL}=20 \mathrm{~mm}$, two main frequencies appear at 3400 and 6800 which have been related to fundamental and harmonic. From the accelerometer measurements (Fig. 7), the second bending mode of the pad was identified by a phase shift between the leading and trailing edges. This phase shift is clearly evidenced for ACL 10, 20 and $30 \mathrm{~mm}$. From the experimental set-up dynamic analysis and disc eigenfrequencies, the $10 \mathrm{~mm}$ ACL configuration seems to involve a mode lock-in between the second bending mode of the pin and the (2-0) disc mode. In the same way mode lock-in between the same pin mode and the (3-0) disc mode seems to occur for the $20 \mathrm{~mm}$ ACL configuration and with the (4-0) disc mode for the $30 \mathrm{~mm}$ configuration. For the $40 \mathrm{~mm}$ ACL configuration any disc mode seems to be involved.

\section{Model of the set-up}

The experimental approach has exhibit possible lock-in between modes of the pin and the disc for some noise occurrences. It has also shown the influence of the pin length even if only 4 ACL have been tested. To improve this investigation, a semi-analytical model has been used. It is based on a minimal model of three degrees of freedom. This model is similar to that used by [22]. The thin plate and pin assembly are described by a two degrees of
Table 3. Parameters used for dynamical analysis.

\begin{tabular}{lll}
\hline$K_{1}(\mathrm{~N} / \mathrm{m})$ & $K_{2}(\mathrm{~N} / \mathrm{m})$ & $\mathrm{D}(\mathrm{mm})$ \\
\hline $256 \times 10^{3}$ & $256 \times 10^{3}$ & 20 \\
\hline
\end{tabular}

freedom system: a translational and a rotational. The disc is modeled as a separate system with only one degree of freedom in the normal direction. This definition is based on the previous experimental results showing out of plane disc modes and the pin ones. The latter corresponds to first and second bending of the pin-plate assembly but the corresponding deformed shapes could be seen as a translation and a tilting of this assembly, which are simulated by the reduced model. To further investigate the dynamic instabilities, a self-excited vibration model with this three degrees of freedom model is proposed as illustrated in Figure 8 .

\subsection{Description of the model}

In the model, $K_{1}$ and $K_{2}$ correspond to the Y-direction stiffness on each side of the thin plate. $y_{1}$ and $y_{2}$ represent the extension of these springs and $d$ is the distance between them. The stiffness of the friction pad is modeled by a parallel distribution of springs $k_{i}$ whose value is directly dependent on the material's mechanical properties (see Sect. 3.2). $y_{i}$ corresponds to the extension of each spring $k_{i}$. Table 3 presents the values used in the computation assuming an elastic behavior constant with the loading intensity.

This analytical model was first computed in static equilibrium under conditions of pressure and sliding. This step rendered it possible to obtain the contact pressure distribution that is injected in a complex modal analysis. The validation of this step is shown in [21]. In the second step of the computation, the complex eigenvalues analysis is performed to determine the natural frequencies of the system. The dynamic equations were as follows:

\section{See this Equation next page.}

In this study, only the out-of-plane bending modes of the disc were considered in accordance with the experimental test. The assumed disc vibration was represented by a model with a single degree of freedom. The equivalent mass $M_{d}$ was set to be equal to the mass of the disc. The disc stiffness $K_{d}$ was calculated using the eigenfrequency determined with the finite element analysis as $M_{d} . M_{p}$ represents the total mass, including the pad and the padhousing, and $J$ is the moment of inertia calculated at the center of mass of the plate spring. The pad-housing and the thin plate were considered to be of steel with mechanical properties set to $210000 \mathrm{MPa}$ for the Young modulus and 0.3 for the Poisson coefficient. All these parameters 


$$
\left\{\begin{array}{l}
M_{d} \ddot{y}_{d}=-K_{d} y_{d}-\sum_{i=1}^{n} k_{i} y_{i} \\
M_{p} \frac{\ddot{y}_{2}-\ddot{y}_{1}}{2}=-K_{1}\left(y_{1}+\frac{y_{2}-y_{1}}{d} x_{1}\right)-K_{2}\left(y_{2}+\frac{y_{2}-y_{1}}{d} x_{2}\right)-\sum_{i=1}^{n} k_{i} y_{i} \\
J \frac{\ddot{y}_{2}-\ddot{y}_{1}}{d}=-K_{1} \frac{y_{2}-y_{1}}{d} x_{1}^{2}-K_{2} \frac{y_{2}-y_{1}}{d} x_{2}^{2}+\sum_{i=1}^{n} k_{i} y_{i} \mu h+\sum_{i=1}^{n} k_{i} y_{i} l
\end{array}\right.
$$

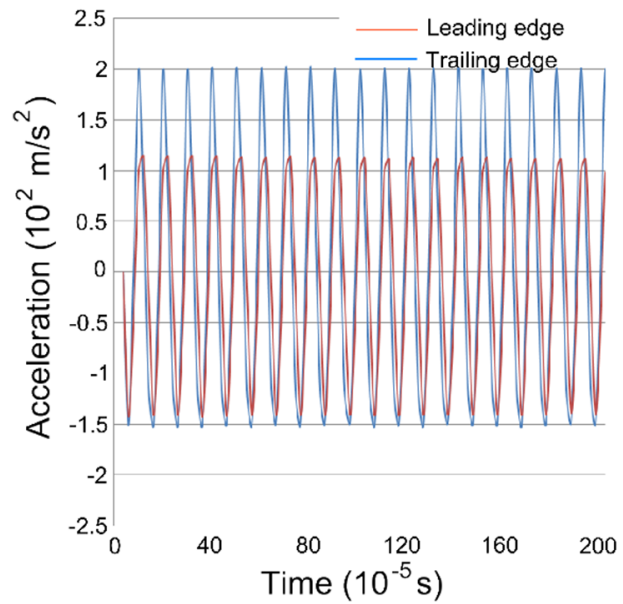

(a)

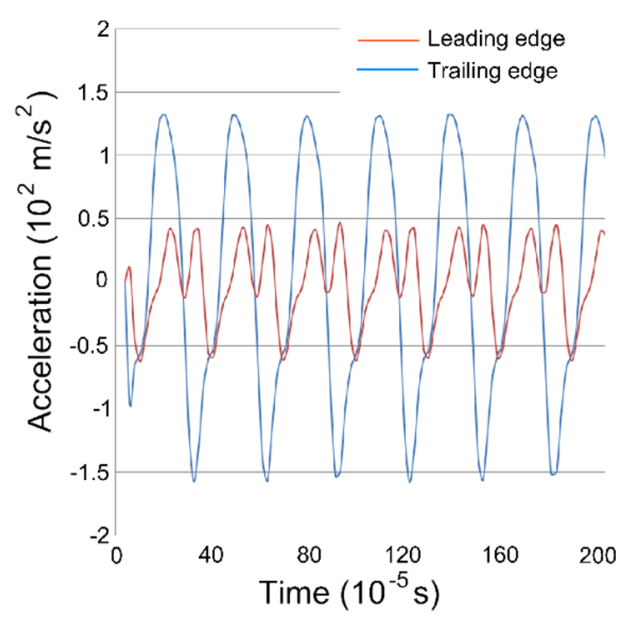

(c)

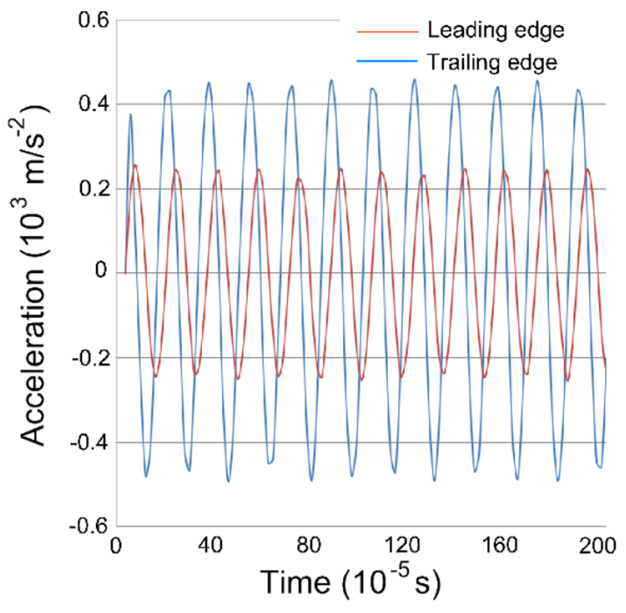

(b)

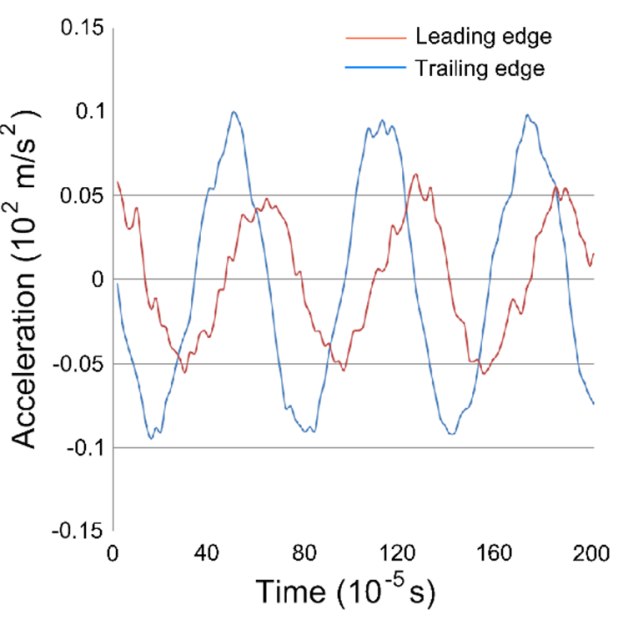

(d)

Fig. 7. Pad acceleration for all ACL configurations (a) $40 \mathrm{~mm}$ (b) $30 \mathrm{~mm}$ (c) $20 \mathrm{~mm}$ (d) $10 \mathrm{~mm}$.

Table 4. Parameters used for dynamical analysis.

\begin{tabular}{lllll}
\hline$M_{d}(\mathrm{~kg})$ & $K_{d}(\mathrm{~N} / \mathrm{m})$ & $M_{p}(\mathrm{~kg})$ & $K_{p}(\mathrm{~N} / \mathrm{m})$ & $\mathrm{J}\left(\mathrm{kg} \cdot \mathrm{m}^{2}\right)$ \\
\hline 3.88 & $4.85 \times 10^{8}$ & 0.07 & $1.17 \times 10^{8}$ & $4.43 \times 10^{-6}$ \\
\hline
\end{tabular}

are presented in Table 4 and were determined from the experimental set-up.

The value of $K_{p}$ is discussed in Section 3.2 its correspond to the linear stiffness of the pad. Finally, $x_{1}$ (resp. $x_{2}$ ) corresponds to the distance between the center of rotation of the pad and the spring $K_{1}\left(\right.$ resp. $\left.K_{2}\right)$. After a quasi-static solution under sliding conditions, a complex eigenvalues analysis was performed and the results were written as $\lambda=\alpha+j \omega$. Here $\omega$ is the eigenfrequency. A positive real part of the complex eigenvalue analysis is being seen as an indication of instability. By examining the real part of the system eigenvalues the modes that are unstable and likely to produce squeal are revealed. The goal of this study is to determine the evolution of the eigenfrequencies of the system according to pad length and material behavior to determine the unstable configurations. These two parameters play an important role for the pressure distribution. 


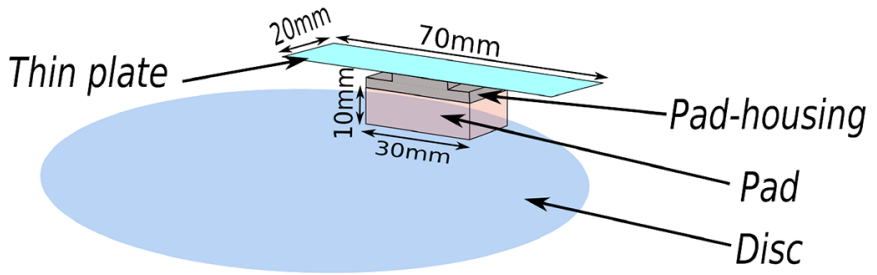

(a)

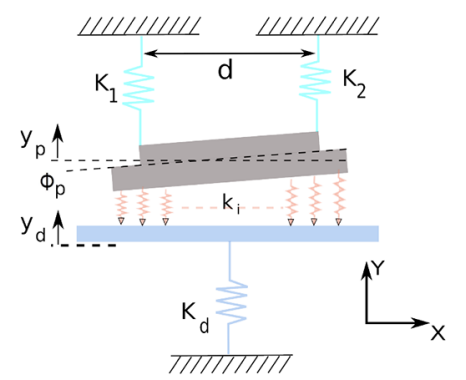

(b)

Fig. 8. Semi-analytic contact model.

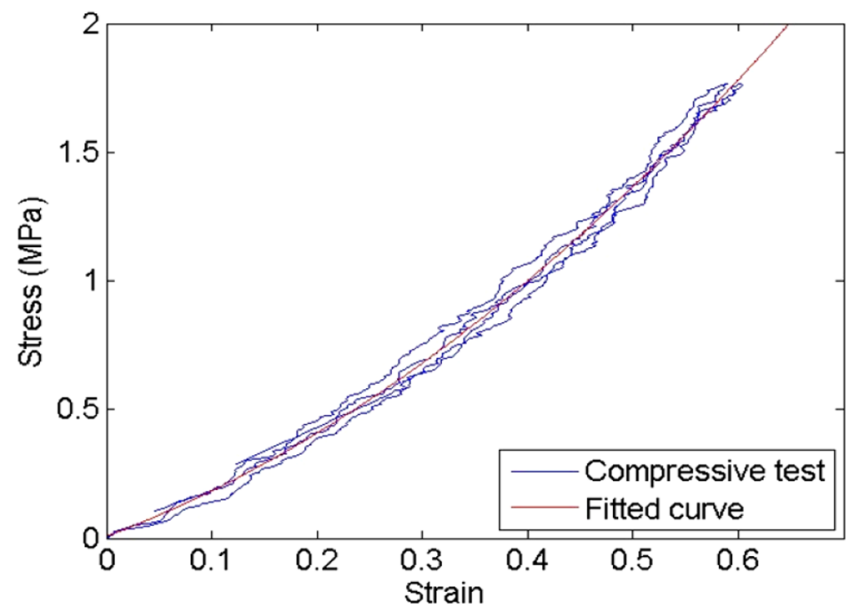

Fig. 9. Experimental results for all apparent contact length configurations.

\subsection{Compressive test}

To identify the material behavior compressive tests have been done. A sample of the friction material with a steel back plate is placed in compressive apparatus. To measure the displacement of compressive plateaus, three Foucault current sensors placed at $120^{\circ}$ has been used. These sensors offer to reduce the uncertainty of measurement due to the wrong parallelism of each face of the pad. The average of sensors has been used to determine the strain calculated by $:=\frac{h-h_{0}}{h_{0}}$ where $h_{0}$ is the initial height of the pad and $h$ the height calculated with the displacement. Stress is calculated by $\sigma=F / S$ where $F$ is measured by load sensor and $S$ is the section of the pad $\left(20 \times 20 \mathrm{~mm}^{2}\right)$. A stress cycle is applied, it is composed by three loadings from 0 to $1.75 \mathrm{MPa}$ and three unloadings.

Figure 9 presents the compressive test results.

Results exhibit a non linear behavior which could be introduced in the relationship between contact stiffness $k_{i}$ and pressure $\mathrm{P}$ based on the red curve. The non linear behavior could be expressed as : $k_{i}=2.3 \times 10^{6} \times$ $P^{(n-1) / n} \mathrm{~N} / \mathrm{m}$ with $n=1.4286$. However a linear behavior could be assumed as often considered in studies of vibrations of frictional systems as brakes. In this case the stiffness $1.17 \times 10^{8} \mathrm{~N} / \mathrm{m}$ is and corresponds to the $K_{p}$ value of Table 4 . It corresponds to a Young modulus of $3000 \mathrm{MPa}$. Note that the material is considered as homogeneous and isotropic.

\section{Results}

This section illustrates how to determine the evolution of the system's eigenfrequencies in relation to the pad length and to identify the unstable configurations. The extremities of the springs $K_{1}$ and $K_{2}$ were submitted to a vertical displacement to obtain an equivalent normal force of $500 \mathrm{~N}$. In the $x$-direction, a translation of the disc was applied to simulate the slip condition. The coefficient of friction is fixed to 0.35 . For the semi-analytical model, the pin contact area was ranged from to $5 \times 20 \mathrm{~mm}^{2}$ to $40 \times 20 \mathrm{~mm}^{2}$. For the pad material properties, two cases are computed. The first one takes into account a constant stiffness corresponding to the linear behavior. The second case considers a non-constant stiffness / non linear behavior / where the value is deduced from the experimental compressive test previously described. The analysis focuses on the influence of the pad contact area which was ranged from to $5 \times 20 \mathrm{~mm}^{2}$ to $40 \times 20 \mathrm{~mm}^{2}$. On the Figure 10, solid lines shows the evolution of eigenfrequencies for linear stiffness and dashed lines for nonlinear stiffness. The red circles represent the experimental squeal frequencies. For each case, the eigenfrequency associated with the translation mode of the disc was slightly modified by the variation of the contact length. On the other hand, the eigenfrequency associated with the tilting (rotation around the $z$ direction) mode of the pad and the translation mode of the pad was strongly affected by the contact surface. Indeed, the frequency of the tilting pad modes increased as the value of the pad length increased. For the calculus with (3-0) disc mode, the tilting mode of the pin and the axial mode of the disc coalesced, leading to an unstable mode, for ACL comprised between 20 and $24 \mathrm{~mm}$ for the constant stiffness case. Including the nonlinear stiffness, the instability range is slightly modified and is obtained for a pin length of $22-27 \mathrm{~mm}$. Note that, for ACL greater than $35 \mathrm{~mm}$, a mode coupling is obtained, involving the two natural modes of the thin plate.

Figure 11 shows a computation of each simulation on the same graph considering the different mode shape $((2,0) ;(3,0) ;(4,0))$ of the disc. In view of these results, four areas of mode lock-in appear with coalescence between two eigenfrequencies (red lines). The comparison between experimental and numerical results shows a good agreement for the identification of the squeal frequencies and the area of instability in term of ACL. The effect of the non-linear behavior of the material modifies the mode coupling for ACL greater than $20 \mathrm{~mm}$. It can also be noticed that the mode lock-in associated with pin length equal to $40 \mathrm{~mm}$ is a confusion of the two pin modes unlike the 


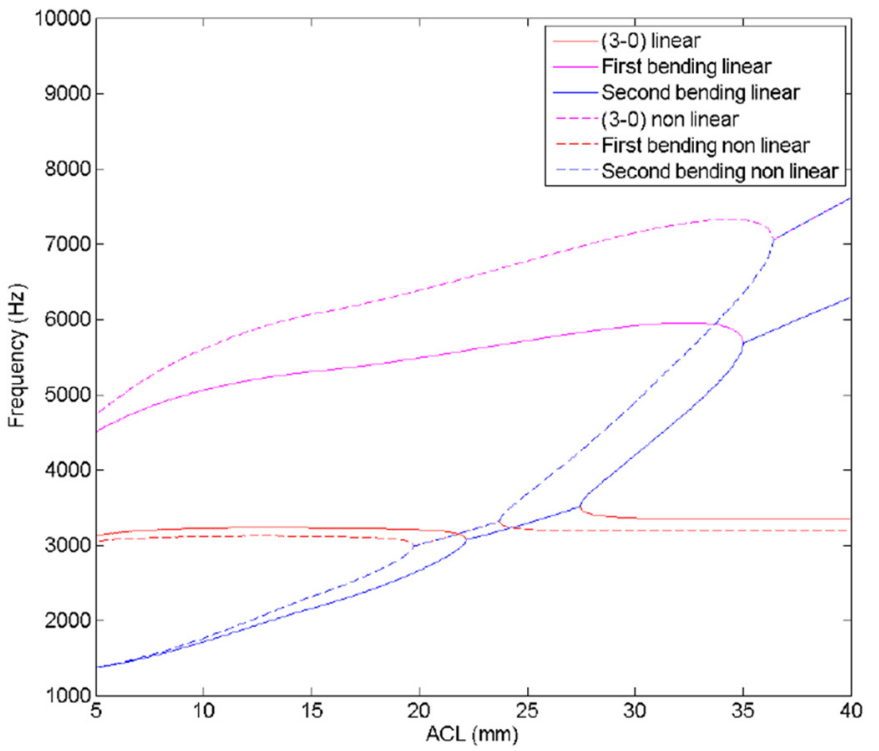

Fig. 10. Semi-analytic contact model for the (3-0) disc mode.

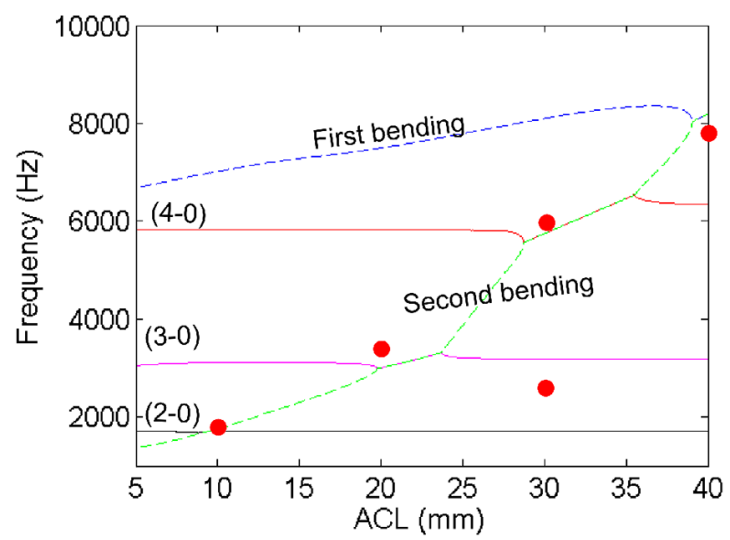

Fig. 11. Evolution of eigenfrequencies of the numerical model (lines) with experimental results (red points) considering each disc mode.

others modes lock-in which are a confusion of the tilting pin mode and a disc mode. These results are also in agreement with the observed deformed shapes of the pad illustrated in Figure 6. These results may be interpreted in terms of brake squeal in the following manner: a change in the brake pin geometry, (adding a chamfer, etc.) may eliminate squeal if the contact length is changed in such a way as to avoid mode coupling. Another conclusion is that significant modifications in contact length lead to another type of mode coupling.

\section{Conclusion}

The aim of this study was to determine the influence of contact conditions, more specifically contact geometry, and friction material behaviour on pin on disc squeal occurrence. Squeal is a complex phenomenon that involves different couplings between the mode shapes of a brake system. The analysis of such a phenomenon was facilitated by using a simplified experimental set-up and a three degrees of freedom model. The influence of contact geometry on squeal occurrence has been clearly shown experimentally. The model permits to investigate the evolution of the eigenfrequencies of the system with the apparent contact length and to clarify the different types of mode coupling results. Two simulations have been performed one with linear behaviour and one with non-linear behaviour. These results are compared with experimental results and good agreement have been obtained especially for non-constant behaviour. The present study only considered homogeneous contact surfaces and low temperatures. However, in practice, brake pads are composed of several components and the friction surface is never entirely smooth $[23,24]$. All these factors can contribute to surface localization. Therefore, the present model may be improved by incorporating discontinuous contact distribution.

The present research work has been supported by the international Campus on Safety and Intermodality in Transportation, the Nord-Pas-de-Calais Region, the European Community, the Regional Delegation for Research and Technology, the Ministry of Higher Education and Research, and the National Center for Scientific Research. The authors gratefully acknowledge the support of these institutions.

\section{References}

[1] A. Akay, Acoustics of friction, J. Acoustic Soc. Am. 111, 1525-1548 (2002)

[2] J.D. Fieldhouse, P. Newcomb, The application of holographic interferometry to the study of disc brake noise, Technical Report SAE, Ref: 930805, 1993

[3] F. Massi, L. Baillet, O. Giannini, Experimental analysis on squeal modal instability, International Modal Analysis Conference - IMAC-XXIV (Missouri), Saint-Louis, USA, 2006

[4] M.R. North, Disc brake squeal - a theorical model. Institute of mechanical engineers, C38/76:169-176, 1976

[5] M. Eriksson, S. Jacobson, Tribological surfaces of organic brake pads, Tribol. Int. 33, 817 (2000)

[6] N. Hoffmann, M. Fischer, R. Allgaier, L. Gaul, A minimal model for studying properties of the modecoupling type instability in friction induced oscillations, Mech. Res. Commun. 29, 197-205 (2002)

[7] Y. Oura, Y. Kurita, Y. Matsumura, Influence of dynamic stiffness in contact region on disk brake squeal, J. Environ. Eng. 4, 234-244 (2009)

[8] F. Bergmann, M. Eriksson, S. Jacobson, The effect of reduced contact area on the occurence of disc brake squeals for automotive brake pad, Proc. Inst. Mech. Eng. 214, 561-568 (2000)

[9] T. Tison, A. Heussaff, F. Massa, I. Turpin, R.F. Nunes, provement in the predictivity of squeal simulations: uncertainty and robustness, J. Sound Vib. 333, 3394-3412 (2014)

[10] N. Hentati, M. Kchaou, A.L. Cristol, D. Najjar, R. Elleuch, Impact of post-curing duration on mechanical, thermal and 
tribological behavior of an organic friction material, Mater. Des. 63, 699-709 (2014)

[11] G. Ostermeyer, On the dynamics of the friction coefficient, Wear 254, 852-858 (2003)

[12] G. Ostermeyer, M. Müller, Dynamic interaction of friction and surface topography in brake systems, Tribol. Int. 39, 370-380 (2006)

[13] H. Ouyang, J.E. Mottershead, M.P. Cartmell, D.J. Brookfield, Friction-induced vibration of an elastic slider on a vibrating disc, Int. J. Mech. Sci. 41, 325-336 (1999)

[14] M. Triches-Jr, S.N.Y. Gerges, R. Jordan, Analysis of brake squeal noise using the finite element method: a parametric study, Appl. Acoust. 69, 147-162 (2008)

[15] Y. Dai, T.C. Lim, Suppression of brake squeal noise applying finite element brake and pad model enhanced by spectral-based assurance criteria, Appl. Acoustics 69, 196-214 (2008)

[16] V. Magnier, D. Naidoo Ramasami, J.F. Brunel, P. Dufrenoy, T. Chancelier, History effect on squeal with a mesoscopic approach to friction materials, Tribol. Int. 115, 600-607 (2017)

[17] Y. Waddad, V. Magnier, P. Dufrénoy, G. De Saxcé, A multiscale method for frictionless contact mechanics of rough surfaces, Tribol. Int. 96, 109-121 (2016)
[18] A.Y. Wang, X.C. Mo, Wang, M.H. Zhu, Z.R. Zhou, Effect of surface roughness on friction-induced noise: exploring the generation of squeal at sliding friction interface, Wear 402403, 80-90 (2018)

[19] E. Denimal, S. Nacivet, L. Nechak, J.J. Sinou, On the influence of multiple contact conditions on brake squeal, Proc. Eng. 199, 3260-3265 (2017)

[20] F. Massi, L. Baillet, O. Giannini, A. Sestieri, Brake squeal: Linear and nonlinear numerical approaches, Mech. Syst. Signal Process. 21, 2374-2393 (2007)

[21] M. Duboc, J.F. Brunel, V. Magnier, P. Dufrénoy, Influence of pin contact geometry and friction material behavior on disc brake squeal noise, NORDTRIB 2014, Aarhus Denmarks, 2014

[22] K. Bonnay, V. Magnier, J.F. Brunel, P. Dufrenoy, G. De Saxce, Influence of geometry imperfections on squeal noise linked to mode lock-in, Int. J. Solids Struct. 75-76, 99-108 (2015)

[23] V. Magnier, E. Roubin, J.B. Colliat, P. Dufrenoy, Methodology of porosity modelling for friction pad: consequence on squeal, Tribol. Int. 109, 78-85 (2016)

[24] Y. Waddad, J.B.V. Magnier, P. Dufrenoy, G. De Saxce, A multiscale method for frictionless contact mechanics of rough surfaces, Tribol. Int. 96, 109-121 (2015)

Cite this article as: M. Duboc, V. Magnier, J.F. Brunel, P. Dufrénoy, Experimental set-up and the associated model for squeal analysis, Mechanics \& Industry 21, 204 (2020) 